\title{
Caffeine Sensitizes U87-MG Human Glioblastoma Cells to Temozolomide through Mitotic Catastrophe by Impeding G2 Arrest
}

\author{
Ning Li $\mathbb{D}$, Pingde Zhang $\mathbb{D}$, Karrie Mei Yee Kiang, \\ Yin Stephen Cheng, and Gilberto Ka Kit Leung \\ Department of Surgery, Li Ka Shing Faculty of Medicine, The University of Hong Kong, Queen Mary Hospital, Hong Kong \\ Correspondence should be addressed to Gilberto Ka Kit Leung; gilberto@hku.hk
}

Received 7 March 2018; Accepted 3 June 2018; Published 28 June 2018

Academic Editor: Monica Fedele

Copyright (C) 2018 Ning Li et al. This is an open access article distributed under the Creative Commons Attribution License, which permits unrestricted use, distribution, and reproduction in any medium, provided the original work is properly cited.

Temozolomide (TMZ) is the first-line chemotherapeutic agent in the treatment of glioblastoma multiforme (GBM). Despite its cytotoxic effect, TMZ also induces cell cycle arrest that may lead to the development of chemoresistance and eventual tumor recurrence. Caffeine, a widely consumed neurostimulant, shows anticancer activities and is reported to work synergistically with cisplatin and camptothecin. The present study aimed to investigate the effects and the mechanisms of action of caffeine used in combination with TMZ in U87-MG GBM cells. As anticipated, TMZ caused DNA damage mediated by the ATM/p53/p21 signaling pathway and induced significant G2 delay. Concurrent treatment with caffeine repressed proliferation and lowered clonogenic capacity on MTT and colony formation assays, respectively. Mechanistic study showed that coadministration of caffeine and TMZ suppressed the phosphorylation of ATM and p53 and downregulated p21 expression, thus releasing DNA-damaged cells from G2 arrest into premature mitosis. Cell cycle analysis demonstrated that the proportion of cells arrested in G2 phase decreased when caffeine was administered together with TMZ; at the same time, the amount of cells with micronucleation and multipolar spindle poles increased, indicative of enhanced mitotic cell death. Pretreatment of cells with caffeine further enhanced mitotic catastrophe development in combined treatment and sensitized cells to apoptosis when followed by TMZ alone. In conclusion, our study demonstrated that caffeine enhanced the efficacy of TMZ through mitotic cell death by impeding ATM/p53/p21-mediated G2 arrest.

\section{Introduction}

Glioblastoma multiforme (GBM) is the most common and aggressive form of primary brain tumor, with an annual incidence of two to three per million adults. It accounts for more than half of all primary intracranial tumors [1]. Surgical removal followed by radiation and chemotherapy is the standard treatment $[2,3]$. However, the recurrence rate remains high, with a median survival of just over one year, rendering GBM one of the most challenging tumors to manage [4].

Temozolomide (TMZ), an oral alkylating agent, is the first-line chemotherapeutics for GBM patients. Its mechanism of action majorly lies in the alkylation of N-7 or O-6 guanine residues or $\mathrm{N}-3$ adenine residues within DNA which leads to mismatches during subsequent DNA replication and consequentially cycle arrest, autophagy, senescence, and cell death [5]. Cell cycle arrest upon DNA damage is thought to be a double-edged sword, however. On the one hand, transient cell cycle delay allows DNA repair and is considered an essential self-protective process in maintaining cellular homeostasis and preventing tumorigenesis in normal tissues. On the other hand, it offers time for tumor cells to erase alkylated residues, correct mismatched base pairs, and eventually promote tumor cell survival, and thereby contributing to chemoresistance and disease recurrence [6]. In this regard, agents that overcome TMZ-induced cell cycle arrest may potentiate its efficacy in GBM treatment.

Caffeine is a widely consumed neurostimulant found in many food products including coffee, tea, and soft drinks. 


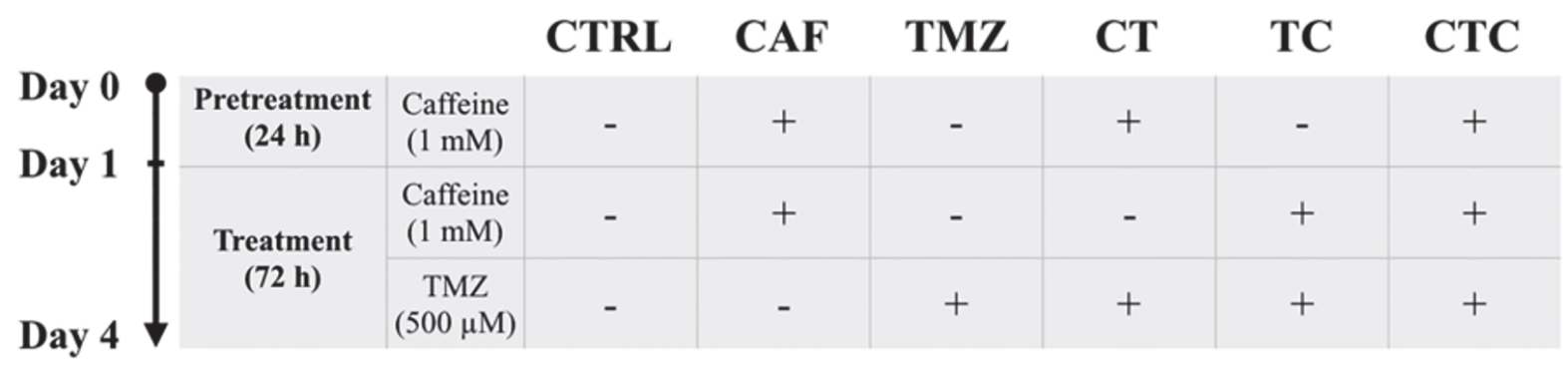

FIGURE 1: Demonstration of experimental strategies. CTRL: control; CAF: caffeine alone; TMZ: temozolomide alone; CT: temezolomide with caffeine pretreatment; TC: combined treatment of temozolomide and caffeine; and CTC: combined therapy with caffeine pretreatment.

Caffeine exerts multiple biological effects such as raising blood pressure [7], affecting gastrointestinal motility [8], and increasing basal metabolic rate [9]. Because it readily penetrates the blood-brain barrier, caffeine can influence psychological performance, enhance long-term memory, and decrease the risk of neurodegenerative diseases such as Parkinson's disease [10]. Recently, an inverse association between caffeine intake and the risk of brain tumors was reported by two epidemiological studies from the United States and Europe [11, 12]. Laboratory evidence further showed that caffeine alone had a suppressive effect on the proliferation of human U87-MG glioma cells in vitro and tumor growth in vivo [13]. Moreover, caffeine reduced the migration of GBM cell lines by impairing focal adhesion complex formation [14]. When used in conjunction with cisplatin or camptothecin, enhanced cytotoxicity against human U251 glioma cells was observed [15]. This synergistic cytotoxicity was attributed to the attenuation of chemotherapy-induced G2 cell cycle delay by caffeine. Against this background, we investigated the effects of TMZ with or without concomitant caffeine on U87-MG human glioma cells in vitro. We also studied the priming effect of caffeine with a view to mimic the real-life situation of high daily caffeine intake prior to the administration of TMZ. The hypotheses were (i) caffeine would enhance TMZ cytotoxicity by attenuating TMZinduced G2 arrest; (ii) pretreatment with caffeine would further enhance this effect.

\section{Materials and Methods}

2.1. Cell Culture and Drug Treatment. Human GBM cell line U87-MG (American Type Tissue Collection, Manassas, VA, USA) was cultured in minimum essential mediumalpha (MEM- $\alpha$ ) supplemented with $10 \%$ heat-inactivated foetal bovine serum (FBS), $100 \mathrm{IU} / \mathrm{ml}$ penicillin, and 100 $\mu \mathrm{g} / \mathrm{ml}$ streptomycin (all from Gibco, Life Technologies, Inc., Carlsbad, CA, USA) in a humidified incubator with $95 \%$ air atmosphere and $5 \%$ carbon dioxide at $37^{\circ} \mathrm{C}$. Caffeine powder was obtained from Sigma, and stock solution was prepared in culture medium at the concentration of 32 $\mathrm{mM}$ and kept in $4^{\circ} \mathrm{C}$. TMZ was obtained from ScheringPlough (Kenilworth, NJ, USA) and dissolved in dimethyl sulfoxide (DMSO, Sigma-Aldrich, Saint Louis, MO, USA) at the concentration $100 \mathrm{mM}$, further diluted in culture medium to $10 \mathrm{mM}$, and stored in $-20^{\circ} \mathrm{C}$. For treatment, caffeine and TMZ were further diluted in medium to the final concentrations as stated below.

A four-day treatment was divided into two phases: oneday pretreatment and a subsequent three-day treatment of either TMZ alone, caffeine alone, or both (Figure 1). Overall, six arms of cells were set up accordingly. The dosage of caffeine was $1 \mathrm{mM}$ as determined by MTT assay, and the dosage of $\mathrm{TMZ}$ we opted was its $\mathrm{IC}_{50}$ value defined in U87MG cells in our previous work [2].

2.2. MTT Assay. This was used to define the experimental dosage of caffeine in this study as well as the efficacy of different treatment strategies. Briefly, cells were plated in 96well microplates at a density of 2000 cells/well overnight for attachment. Caffeine with different concentrations ranging from 0.05 to $10 \mathrm{mM}$ was administered on the second day, and cells were incubated for $96 \mathrm{~h}$. On the fourth day, $10 \mu \mathrm{l}$ MTT stocking solution ( $5 \mathrm{mg} / \mathrm{ml}$, Sigma-Aldrich) was added on the top of the culture medium, and cells were lysed by adding $100 \mu \mathrm{l}$ DMSO. Absorbance was acquired at $595 \mathrm{~nm}$ using spectrophotometer (MultiSkan FC, Thermo Scientific).

To examine the combined effects of caffeine and TMZ on viability, cells were seeded at a $2 \times 10^{4} /$ well in 6well plates for microscopic imaging or $2 \times 10^{3} /$ well in 96well culture plates for MTT assay, respectively. After full attachment, cells were treated with six different strategies as stated. For microscopic observation, cells in 6-well plates were washed with $1 x$ phosphate buffered saline (PBS) and fixed by $4 \%$ paraformaldehyde for image taking. MTT assay was conducted to test the cytotoxicity of different treatment as previously described. Three experiments were performed separately in quadruplicates.

2.3. Colony Formation Assay. For longer-term observation, a clonogenic assay was performed. Briefly, 100 cells/well were seeded in 6-well plates and incubated overnight to allow full attachment. On the following four days, different treatment strategies were applied as stated above except that the concentration of TMZ was reduced from $500 \mu \mathrm{M}$ to 50 $\mu \mathrm{M}$. All cells were released free on the fifth day and allowed 
to be maintained in fresh medium for another 10 days. Then cells were fixed with $75 \%$ ethanol and stained with crystal violet solution ( $5 \mathrm{~g} / \mathrm{L}$, Sigma-Aldrich). Colonies comprising more than fifty cells in each well were counted and analyzed under microscopy.

2.4. Cell Cycle Analysis. Cells were harvested and washed with PBS and fixed in $70 \%$ ethanol at $4^{\circ} \mathrm{C}$ overnight. On the following day, cells were washed twice with PBS and resuspended in staining solution containing $20 \mu \mathrm{g} / \mathrm{ml}$ propidium iodide and $100 \mu \mathrm{g} / \mathrm{ml}$ RNase (both from Thermo Fisher Scientific Inc., Waltham, MA) in PBS. After maintaining the reaction in the dark at $37^{\circ} \mathrm{C}$ for $60 \mathrm{~min}$, cells were washed and resuspended in PBS before analysis on BD FACSCalibur flow cytometry; results were analyzed using FlowJo software.

2.5. Immunoblotting. Treated cells were washed with ice-cold PBS twice and collected for homogenization with lysis buffer (Cell Signaling, Beverly, MA) containing proteinase inhibitor cocktail (Roche Diagnostics, Indianapolis, IN, USA) to obtain total cellular protein. Protein samples (20-25 $\mu \mathrm{g}$ per lane) were electrophoresed on a $10 \%$ or $12 \%$ sodium dodecyl sulfate polyacrylamide gel and transferred to polyvinylidene fluoride membranes. After blocking in 5\% non-fat milk for $1 \mathrm{~h}$, membranes were incubated with primary antibodies including p-ATM (1:1000), p53 (1:1000), p-p53 (1:1000), p21 Waf1/Cip1 (p21, 1:1000), caspase-3 (1:1000), cleaved caspase-3 (1:500), and GAPDH (1:2000) (all from Cell Signaling) overnight at $4^{\circ} \mathrm{C}$. On the following day, anti-rabbit (for p-ATM, p-p53, p21, caspase-3, cleaved caspase-3 and GAPDH, 1:10000, Santa Cruz, CA, USA) or anti-mouse (for p53, 1:50000, SigmaAldrich) peroxidase-conjugated secondary antibodies. Protein bands were detected with chemiluminescent reagents (GE Healthcare, Buckinghamshire, UK) and then exposed to $\mathrm{X}$-ray film.

2.6. Immunofluorescent Staining. Cells were seeded on 12 $\mathrm{mm}$ coverslips and incubated with corresponding treatments. On the harvest day, cells were fixed with $-20^{\circ} \mathrm{C}$ methanol for $5 \mathrm{~min}$ at room temperature. Cells were then incubated with $10 \%$ normal goat serum (Dako Corp., Carpinteria, CA) for $1 \mathrm{~h}$, followed by incubation with $\alpha$-tubulin primary antibody (1:200, Santa Cruz) overnight at $4^{\circ} \mathrm{C}$. A fluorescein-conjugated secondary antibody (Thermo Fisher Scientific) was used for visualization of the signal. After $1 \mathrm{~h}$ of incubation, DAPI counterstaining was performed, and cell counting and the image obtaining were conducted under the fluorescent microscope. The entire procedure was performed under light-proof conditions.

2.7. Cell Counting and Statistical Analysis. For cell counting, twenty random fields for each sample were chosen. All positive cells with multiple spinal poles or micronuclei were counted and summed up as the final number for statistical analysis. The number of cells was quantified by using Image J (version 1.50; NIH, Bethesda, MA, USA) in a blinded manner.

All data in the text were expressed as mean \pm SD, and statistical analyses were performed using Prism 6 and SPSS

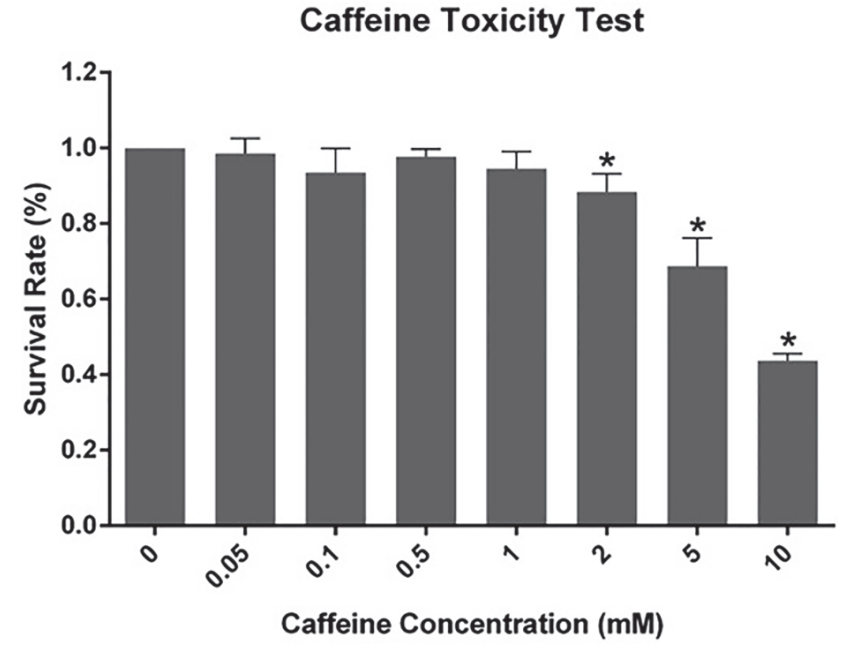

FIgURE 2: MTT results for caffeine toxicity. Dosages below $1 \mathrm{mM}$ did not affect the viability of U87-MG cells. Therefore, $1 \mathrm{mM}$ was chosen as the target dosage in the further experiments. $* p<0.05$ compared to control.

19.0.0. Multiple t-test or one-way ANOVAs with Tukey's multiple comparisons test were performed to evaluate differences among groups. A $p$ value $<0.05$ was considered statistically significant.

\section{Results}

3.1. Caffeine Enhances TMZ's Chemoefficacy in Both Shortand Long-Term Observations. As shown in Figure 2, cell viability was not significantly affected by caffeine alone below a dosage of $1 \mathrm{mM}$ during a four-day course; $1 \mathrm{mM}$ was therefore selected for use in subsequent experiments. Threeday incubation with $500 \mu \mathrm{M}$ TMZ reduced cell proliferation to $50 \%$, with a further $12 \%$ reduction after cotreatment with caffeine (TMZ versus TC, $p<0.05$, Figure $3(\mathrm{~b})$ ). Interestingly, pretreatment with caffeine for one day in advance further enhanced the efficacy of cotreatment (TC versus CTC, $p$ $<0.05)$. Though a slightly reduced cell survival was also observed in CT cells (i.e., caffeine followed by TMZ alone), no statistical difference was reached when compared with that of TMZ alone (CT versus TMZ, $p>0.05$ ). Microscopic observations concurred with MTT assays and revealed the same trend (Figure 3(a)).

On colony formation assay, $50 \mu \mathrm{M}$ TMZ alone resulted in an obvious decrease in the number of colonies in comparison with control, and preexposure to caffeine did not bring additional benefits (TMZ versus CT, $p>0.05$ ). Concomitant treatment with TMZ and caffeine (i.e., TC group) reduced the number of colonies to $36 \%$ of those in CTRL and $58 \%$ of those in the TMZ alone group, suggesting an enhanced antiproliferative effect with the use of caffeine. In line with the MTT results, cell growth was further inhibited if cells were given caffeine one day before combined therapy (Figures 3(c) and $3(\mathrm{~d}))$. Altogether, these data suggested that caffeine could enhance TMZ cytotoxicity in both short and long terms. 


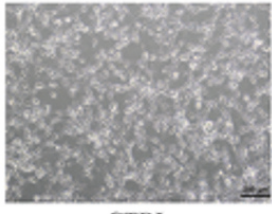

CTRL

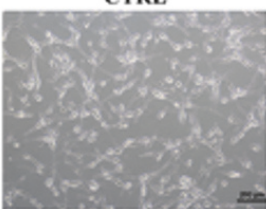

CT

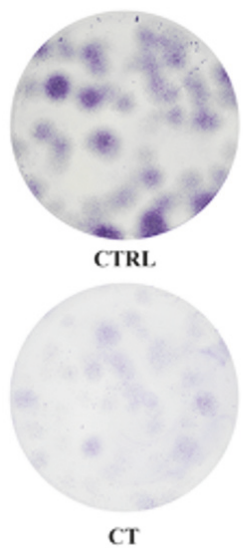

CT

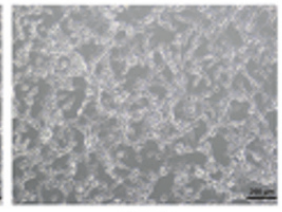

CAF

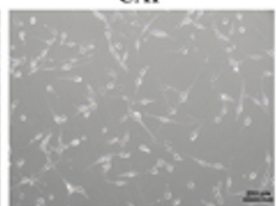

TC

(a)

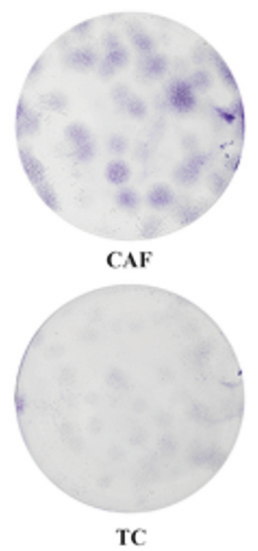

(c)

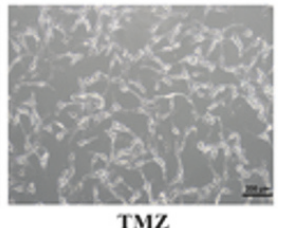

TMZ

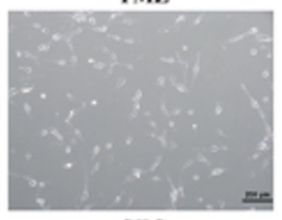

CTC

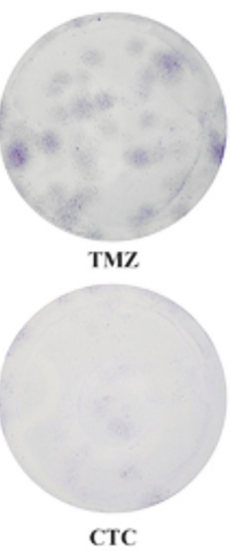

CTC

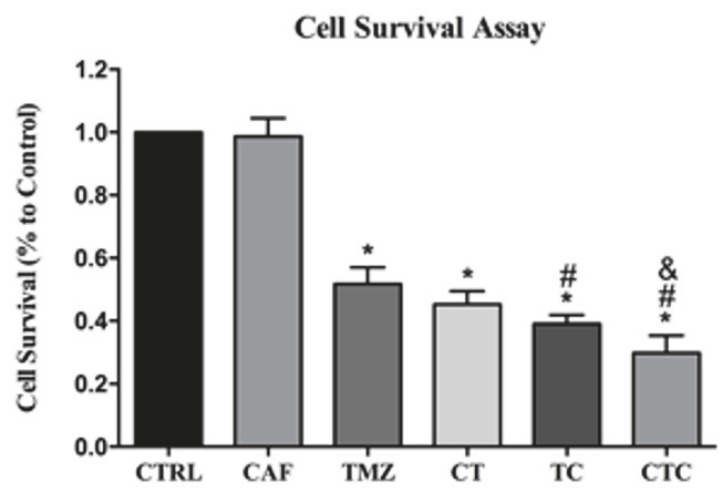

(b)

Colony Formation Assay

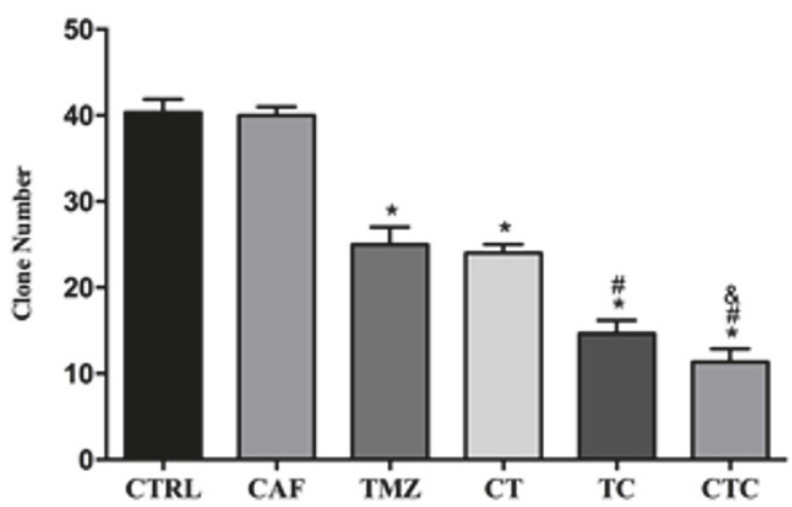

(d)

FIGURE 3: Results of MTT (b) and colony formation assay (c,d). Both assays showed that combination of caffeine and TMZ exerted enhanced antiproliferative effect in comparison with TMZ alone. Furthermore, pretreatment of caffeine produced additional benefits only when it was followed by combined treatment but not TMZ alone. Images from bright field demonstrated the same trend (a). *, \#, and \& represent for $p<$ 0.05 when compared to control, TMZ, and TC, respectively.

\subsection{Caffeine Abrogates TMZ-Induced G2/M Arrest through} Inhibiting ATM-p53-p21 Pathway. TMZ causes DNA damage, triggers repair responses, and induces significant cell cycle arrest in glioma cell lines; attenuating this cell cycle delay may facilitate chemocytotoxicity [16]. Caffeine is known as a potent cell cycle modulator by regulating ATM-mediated signaling pathway [17]. Hence, we next asked whether or not this may explain the enhanced chemoefficacy as described above. Flow cytometry results showed that TMZ alone produced a dramatic cycle delay with more than half of the cells being trapped in G2 phase (Figure 4). The presence of caffeine together with TMZ partially abrogated the G2 arrest, as the number of arrested cells decreased by approximately $30 \%$ when compared to that observed after TMZ-alone treatment. Meanwhile, the proportion of cells with multinucleation $(\mathrm{MN})$ increased with concomitant caffeine (TMZ versus TC, $p<0.05)$. MN or polyploid peak was usually considered to be a marker of improper cell divisions, characterized by an increased amount of cells containing multiple sets of chromosomes [18]. The obvious increase of MN peak indicated that cotreatment with TMZ and caffeine induced the polyploid formation in U87-MG cells. The results were also in line with the aforementioned findings that the largest amount of cells with MN was seen when caffeine was given both before and during TMZ treatment. Accompanying these results, we also witnessed a sub-G1 peak in four TMZ-treated groups, indicating that TMZ caused apoptotic cell death. Combined treatment again showed a larger amount of cells located in sub-G1 area; however, caffeine pretreatment did not bring additional increase of sub-G1 population, be it followed by TMZ alone or combined treatment (further discussed below).

Immunoblotting results showed marked ATM phosphorylation in response to TMZ challenge, which concurrently led to an activation of its downstream p53 and p21. Combined treatment with TMZ and caffeine suppressed the activation of ATM/p53/p21 pathway; the phosphorylation of ATM and p53 was restrained, and the expression of p21 was downregulated (TMZ versus TC). Moreover, this effect was further augmented when caffeine was given $24 \mathrm{~h}$ ahead of TMZ plus caffeine (TC versus CTC) (Figure 5).

3.3. Caffeine Induced Cell Death through Mitotic Catastrophe, Which May Be Independent from Apoptosis. When cells with 

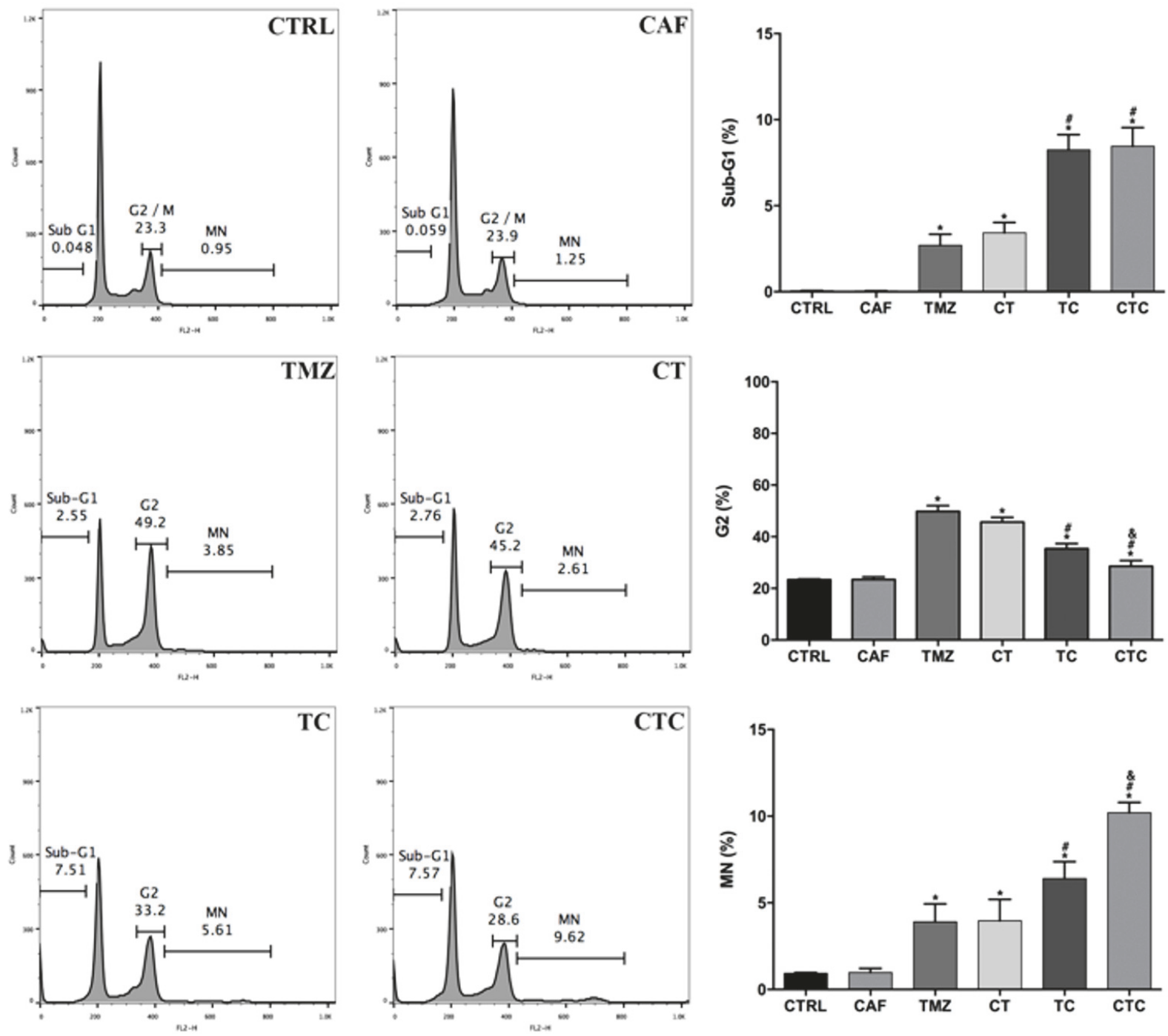

FIGURE 4: Representative cell cycle analysis in different treatment strategies. Combination of caffeine and TMZ impeded TMZ-induced G2 delay in U87-MG cells and caused an increase in the sub-G1 and MN phase (TMZ versus TC/CTC, $p<0.05$ ). An additional increase in MN proportion but not sub-G1 was seen in pretreatment plus combination strategy. *, \#, and \& represent for $p<0.05$ when compared to control, TMZ, and TC, respectively.

damaged DNA bypass the cell cycle checkpoint and enter the mitotic phase, they may manifest mitotic catastrophe (MC), which is defined as a mechanism of mitosis-linked cell death due to inappropriate entry into mitosis [19]. In the present study, two characteristic features of MC, micronucleation and mitotic spindle disruptions, were determined. We found that the majority of nuclei were oval-shaped in CTRL cells, while cells with multiple nucleic fragments were observed in TMZtreated populations (Figure 6(a), DAPI staining). It was more frequently seen in combined treatment (TC) and could be further enhanced when pretreatment was introduced (CTC, Figure 6(b)). Aberrant chromosome segregation represented as multiple spindle poles is also a typical feature of MC. In our studies, over $8 \%$ of cells showed multiple spindle poles which indicated mis-segregation of chromosomes in TMZ group; this compared with only around 3\% found in
CTRL and CAF (Figures 6(a) and 6(b), DAPI and $\alpha$-tubulin counter staining). Then, the number of cells going through multipolar spindle poles doubled when TMZ was combined with caffeine. Consistent with our cell cycle analysis, pretreatment with caffeine only produced additional effect when followed by combined therapy but not TMZ alone, as there were $60 \%$ more cells suffering from chromosome missegregation in CTC in comparison with TC. These results suggested that combined therapy brought more significant disturbances to chromosome segregation and mitosis that would be indicative of an essential involvement of MC.

We then further investigated the level of cleaved caspase3 , an apoptosis marker. TMZ induced caspase-3 cleavage, which was augmented when cells were preexposed to caffeine (Figures 6(c) and 6(d)). Concurrent treatment then showed a more powerful induction in caspase-dependent apoptosis, 

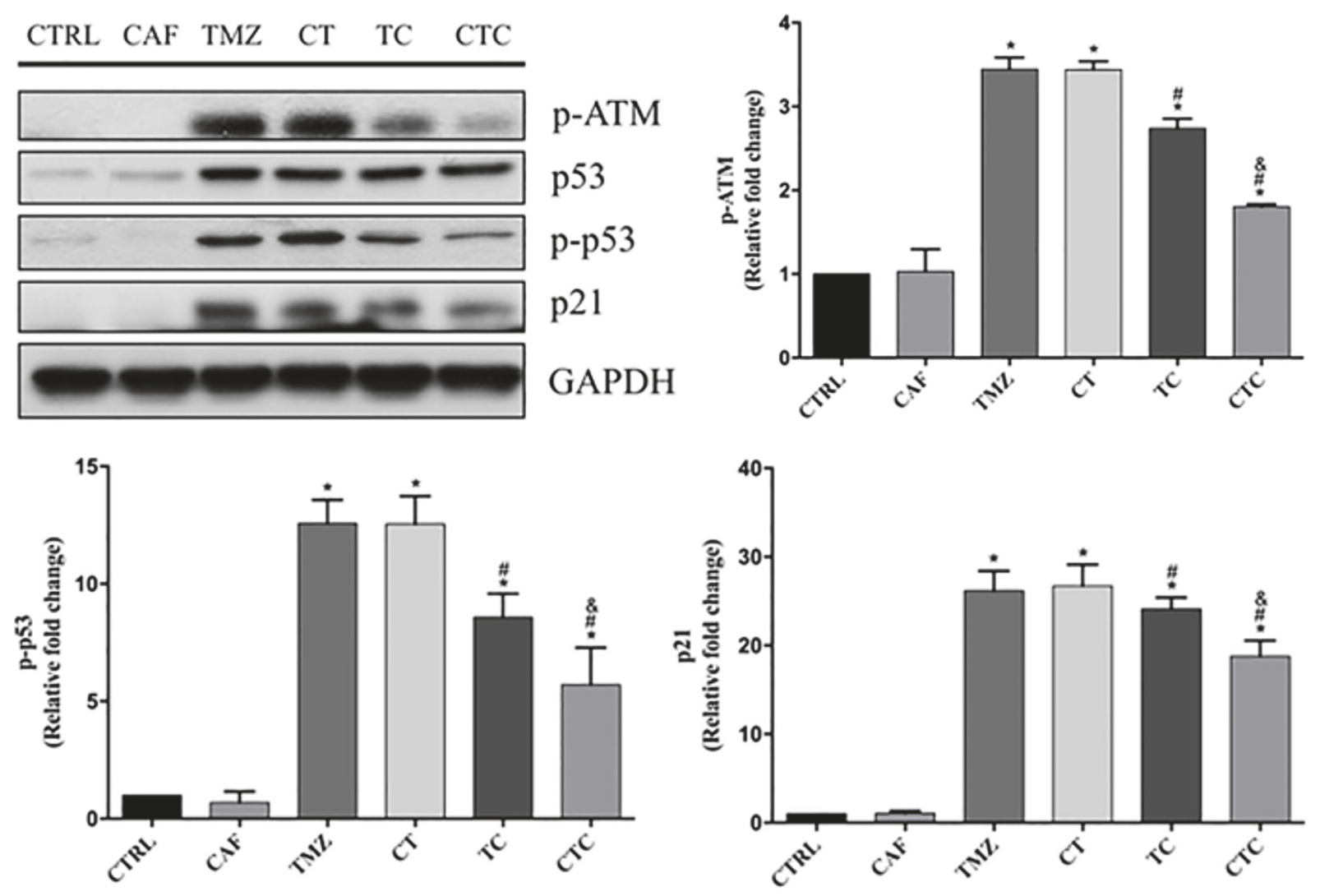

FIGURE 5: Immunoblotting analysis showed that TMZ-induced activation of ATM/p53/p21 pathway was repressed by concurrent caffeine (TMZ versus TC), and it was further inhibited by preexposure of caffeine $24 \mathrm{~h}$ ahead of combined therapy (TC versus CTC). GAPDH was used as an internal loading control. *, \#, and \& represent for $p<0.05$ when compared to control, TMZ, and TC, respectively.

while pretreatment did not bring more benefits in this case. These findings were inconsistent with the MC alterations, indicating that caffeine could also work as an apoptotic synergist with $\mathrm{TMZ}$ independently from MC. Indeed, apoptosis by sub-G1 (Figure 4) did not show a clear association between apoptosis and MC either, and that no difference was observed between TC and CTC.

\section{Discussion}

Recent epidemiological studies demonstrated the benefits of caffeinated drink intake in decreasing the risk of brain oncogenesis [11, 12]; caffeine alone was also reported to suppress the proliferation and migration of GBM cells both in vitro and in vivo $[13,14]$. Caffeine's synergistic effects with radiotherapy and chemotherapy were also demonstrated in adenocarcinoma cells [20], hepatocellular carcinoma cells [21], and cervical carcinoma cells [22]. In GBM, Janss et al. found that caffeine was a potent sensitizer for cisplatin and camptothecin, as it enhanced the cytotoxicity of both drugs in U251 cells [15]. In line with these findings, our results demonstrated that caffeine at a noncytotoxic concentration promoted the efficacy of TMZ in U87-MG cells. This chemosensitizing benefit produced by concurrent caffeine treatment was likely related to its regulation of cell cycle progressions. It has been known that cell cycle delay upon checkpoint activation following DNA damaging treatments such as TMZ can facilitate DNA repairs, promote cancer cells survival, and lead to chemoresistance [6]. These dormant cells may later exit cell cycle temporarily while remaining metabolically active and are also thought to be more resistant to chemotherapy when they reenter the cell cycle and begin to divide again after a period of time [20]. In the case of GBM, TMZ treatment produces marked cell cycle arrest which could partially explain the high rate of TMZ tolerance and disease relapse in clinical treatment [23]. Our previous works using TMZ-resistant U87-MG and D54-MG lines also revealed dramatic cell cycle arrest after long-term TMZ exposure, indicating that a close association indeed exists between cell cycle delays and the onset of TMZ resistance [24]. Conversely, the attenuation of this cell cycle arrest may potentially promote the efficacy of current chemotherapies and reduce the incidence of chemoresistant relapse.

ATM is one of the essential DNA damage response (DDR) kinases, and its activation can restore genomic integrity in response to DNA instability involved in a variety of cellular processes [25]. Activation of ATM leads to subsequent phosphorylation of downstream substrates, such as p53 and p21, and exerts its effects on DNA repair, cell death, and, most importantly, cell cycle arrest [26]. Therefore, its activation in GBM following TMZ treatment is considered to be responsible for the cell cycle delay due to DNA 


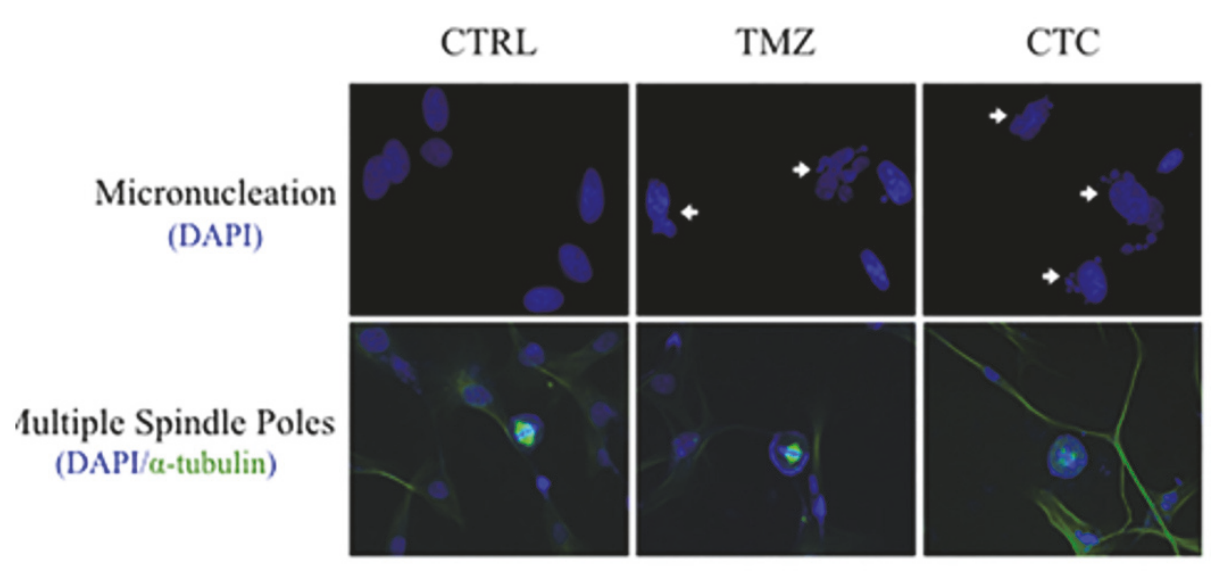

(a)

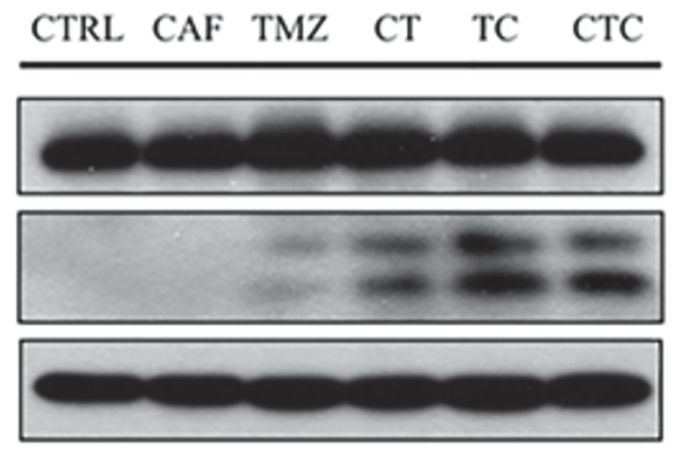

(c) caspase-3

cl. caspase-3

GAPDH

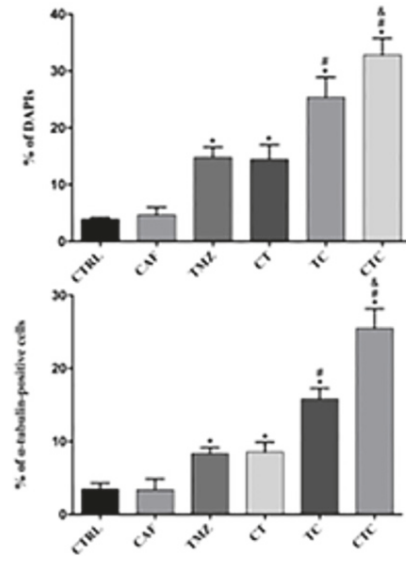

(b)

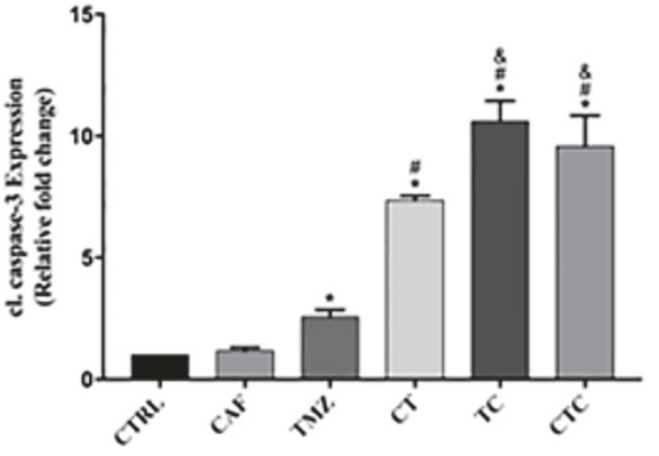

(d)

FIGURE 6: Representative immunofluorescent images illustrating characteristic micronucleation and multiple spindle poles during MC occurrence (a). Proportion of cells showing MC features increased after TMZ treatment, which could be enhanced by concurrent exploitation of caffeine. Pretreatment of caffeine only exerted additional effect when it was followed by combined treatment but not TMZ alone. *, \#, and $\&$ represent for $p<0.05$ when compared to control, TMZ, and TC, respectively (b). While combination of caffeine augmented TMZ-induced caspase-3 cleavage, early exposure of caffeine only produced additional benefits to TMZ but not combined treatment. *, \#, and \& represent for $p<0.05$ when compared to control, TMZ, and CT, respectively (c, d).

double strand breaks (DSBs). Pharmacological inhibition of ATM reverses this G2 arrest and renders GBM cells more susceptible to TMZ [27]. In the present study, TMZ dramatically increased the phosphorylation of ATM and its important downstream factors p53 and p21 as anticipated, leading to a substantial G2 stage delay. Caffeine is known as an inhibitor of ATM kinase activity [17], and cotreatment of caffeine suppressed the activation of ATM signaling pathway and ameliorated TMZ-induced G2 arrest, which contributed to the augmentation of TMZ chemoefficacy. These findings are in agreement with previous studies in which caffeine augmented radiotherapeutic benefits by inhibiting ATM and ATR activities in lung adenocarcinoma cells and leukemia cells [28].

We also observed an inhibition of phosphorylation of p53, a well-known tumor suppressor, through the suppression of ATM activation in combined TMZ plus caffeine treatment. Extensive studies had shown correlations between p53 functional loss and tumorigenesis of liver cancer, lung cancer, colon cancer, and GBM [29]. Activation of p53 plays a crucial role in TMZ therapies in GBM treatment as it is believed to mediate apoptotic cell death following ATM-mediated DDR [30]. In this regard, one might expect that downregulating p53 would work against DNA damaging agents in the treatment of GBM. However, current evidence suggests that p53 participation in anticancer therapy exhibits a two-armed effect; in addition to apoptosis, p53 mediates checkpoint activation and induces cell cycle delay which is considered to favor DNA repair and attenuate chemoefficacy [31]. In the present study, although the phosphorylation of p53 was significantly suppressed, chemoefficacy was augmented as both mitotic cell death and apoptosis were enhanced following treatment with both caffeine and TMZ (TMZ versus TC). This result agreed with several works which demonstrated that GBM cells with inactive p53 were more sensitive to TMZ treatment than those with wild-type p53 [32,33].

We surmised, and our findings suggested, that GBM cells that reenter cell cycle following our treatments would carry impaired DNA and experience aberrant and incomplete mitosis. The latter may lead to cell division failure and cellular break down. This form of catastrophic cell division, or MC, is characterized by the distinguishing features of giant cells 
with micronuclei formation and multiple spindle poles, both of which reflect an abnormal segregation of chromosomes [34]. G2 arrest prevents MC by halting premature entry, and MC will manifest when G2 arrest is abrogated. In this work, we observed an inverse association between the extent of G2 arrest and frequency of MC. The number of cells showing large cell bodies with micronucleation and multipolar spindles was larger in combined treatment groups when compared with TMZ alone. This trend was in line with the alterations in cell cycle analysis where G2 peak was lower and MN peak was higher in TC and CTC groups in comparison with TMZ alone. Our results supported the notion that caffeine produced its chemosensitizing effects by promoting mitotic cell death through abrogating TMZinduced G2 delay.

So far, there is no general consensus on the relationship between MC and apoptosis [35]. Caspases were reported to be essential for the termination of $\mathrm{MC}$, indicating that premature mitotic failure might act as an intermediate process leading to ultimate apoptotic cell death $[36,37]$. On the other hand, in p53-deficient U-2 OS bone osteosarcoma cells, MC could be induced by adeno-associated virus in the absence of caspase activation and apoptosis [38]. In another MDR1-induction model in HeLa cells, apoptosis was significantly suppressed, while cellular fractions resulted from MC increased after ionizing radiation [39]. The obvious phenotypic differences between apoptosis and MC also supported the notion that these two cellular processes may be independent. Herein, although combined treatment enhanced both apoptosis and MC, early caffeine exposure only brought additional benefits to apoptotic cell death when followed by TMZ alone, which was in contrary with its effect on MC development. Supportively, a previous study using SH-SY5Y human neuroblastoma cell line showed that pretreatment with caffeine increased the sensitivity of cells to doxorubicin-induced apoptosis secondary to an increased production of mitochondrial-free radical [40]. Together with our data, we propose that caffeine pretreatment before the administration of chemotherapeutics could sensitize glioma cells to apoptosis that is likely independent from MC.

Last but not least, it was interesting to observe that caffeine-pretreated cells showed an enhanced sensitivity to combined treatment through MC augmentation but not to TMZ alone. It has been reported that long and chronic caffeine exposure would sensitize organisms to some sort of drugs such as methylphenidate [41], but the mechanism is far from clear. In the study by Susan et al., although the caffeine-induced sensitization of neuroblastoma cells to doxorubicin was attributed to increased mitochondrial-free radical production, a decrease of total reactive oxygen species (ROS) production was also noticed [40]. Evasion of ROS production by antioxidants was reported to facilitate MC development [42], indicative of a potential involvement of ROS regulation in a setting similar to our study. Note should be taken that potentiation by caffeine to chemotherapy is cell line- and agent-specific [40]; therefore, further studies in other GBM cell lines as well as in animal models are needed.

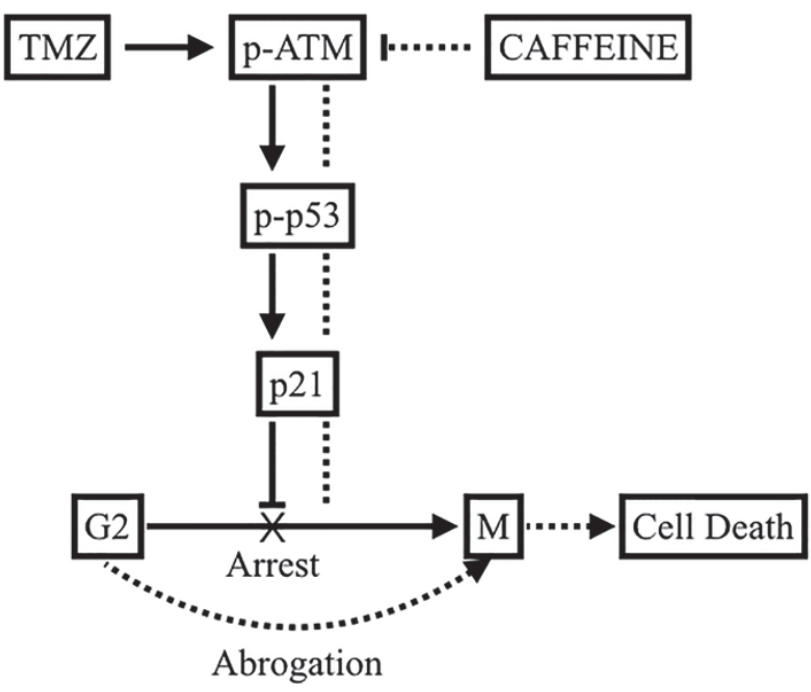

FIGURE 7: Proposed mechanisms for caffeine in sensitizing TMZ's efficacy. TMZ causes DNA damage, activates ATM/p53/p21 pathway, and induces significant G2 arrest (full line). Caffeine inhibits the phosphorylation of ATM and thus suppresses the activation of DDR pathway. G2 delay is abrogated, cells with damaged DNA enter mitotic phase prematurely, and augmented MC occurs (dotted line). Our results did not show a dependent association between MC and apoptosis; hence further studies are warranted.

\section{Conclusions}

The present study demonstrated that caffeine enhanced TMZ's chemoefficacy through impeding G2 delay by inhibiting ATM/p53/p21 pathway and the promotion of mitotic catastrophe (Figure 7). Our results highlighted the following. (1) Caution should be paid to the fact that cancer cells possess different intrinsic responses to chemotherapies, and GBM is known to be resistant to apoptosis. TMZ induces significant cell cycle arrest at an early stage of treatment rather than cell death, which could account for the frequent clinical relapses seen after TMZ treatment. (2) Attenuating G2 delay after TMZ treatment enhances MC, which may then lead to cellular breakdown in ways that may be either dependent or independent from apoptosis. This particular property should be further explored in the treatment of GBM. (3) Caffeine pretreatment augments the sensitizing efficacy of combined treatment, indicating the potential benefit of a high intake of caffeinated products before and during TMZ treatment. In vivo confirmation of our findings as well as epidemiological studies is needed.

\section{Data Availability}

The data used to support the findings of this study are available from the corresponding author upon request.

\section{Conflicts of Interest}

The authors declare no conflicts of interest regarding the publication of this paper. 


\section{References}

[1] K. Urbańska, J. Sokołowska, M. Szmidt, and P. Sysa, "Review Glioblastoma multiforme - an overview," Współczesna Onkologia, vol. 5, pp. 307-312, 2014.

[2] P. Zhang, S. Sun, N. Li et al., "Rutin increases the cytotoxicity of temozolomide in glioblastoma via autophagy inhibition," Journal of Neuro-Oncology, vol. 132, no. 3, pp. 393-400, 2017.

[3] R. M. Young, A. Jamshidi, G. Davis, and J. H. Sherman, "Current trends in the surgical management and treatment of adult glioblastoma," Annals of Translational Medicine, vol. 3, p. 121, 2015.

[4] A. M. Rulseh, J. Keller, J. Klener et al., "Long-term survival of patients suffering from glioblastoma multiforme treated with tumor-treating fields," World Journal of Surgical Oncology, vol. 10, article 220, 2012.

[5] J. Zhang, M. F. G. Stevens, and T. D. Bradshaw, "Temozolomide: Mechanisms of action, repair and resistance," Current Molecular Pharmacology, vol. 5, no. 1, pp. 102-114, 2012.

[6] W. P. Roos, A. D. Thomas, and B. Kaina, "DNA damage and the balance between survival and death in cancer biology," Nature Reviews Cancer, vol. 16, no. 1, pp. 20-33, 2016.

[7] J. W. Daniels, P. A. Molé, J. D. Shaffrath, and C. L. Stebbins, "Effects of caffeine on blood pressure, heart rate, and forearm blood flow during dynamic leg exercise," Journal of Applied Physiology, vol. 85, no. 1, pp. 154-159, 1998.

[8] P. J. Boekema, M. Samsom, G. P. van Berge Henegouwen, and A. J. Smout, "Coffee and gastrointestinal function: Facts and fiction: A review," Scandinavian Journal of Gastroenterology, vol. 34, no. 230, pp. 35-39, 1999.

[9] P. Koot and P. Deurenberg, "Comparison of changes in energy expenditure and body temperatures after caffeine consumption," Annals of Nutrition and Metabolism, vol. 39, no. 3, pp. 135142, 1995.

[10] A. Ascherio, S. M. Zhang, M. A. Hernán et al., "Prospective study of caffeine consumption and risk of Parkinson's disease in men and women," Annals of Neurology, vol. 50, no. 1, pp. 56-63, 2001.

[11] C. N. Holick, S. G. Smith, E. Giovannucci, and D. S. Michaud, "Coffee, tea, caffeine intake, and risk of adult glioma in three prospective cohort studies," Cancer Epidemiology, Biomarkers \& Prevention, vol. 19, no. 1, pp. 39-47, 2010.

[12] D. S. Michaud, V. Gallo, B. Schlehofer et al., "Coffee and tea intake and risk of brain tumors in the European Prospective Investigation into Cancer and Nutrition (EPIC) cohort study," American Journal of Clinical Nutrition, vol. 92, no. 5, pp. 11451150, 2010.

[13] B. M. Ku, Y. K. Lee, J. Y. Jeong et al., "Caffeine inhibits cell proliferation and regulates PKA/GSK $3 \beta$ pathways in U87MG human glioma cells," Molecules and Cells, vol. 31, no. 3, pp. 275279, 2011.

[14] Y. Chen, W.-C. Chou, Y.-M. Ding, and Y.-C. Wu, "Caffeine inhibits migration in glioma cells through the ROCK-FAK pathway," Cellular Physiology and Biochemistry, vol. 33, no. 6, pp. 1888-1898, 2014.

[15] A. J. Janss, C. Levow, E. J. Bernhard et al., "Caffeine and staurosporine enhance the cytotoxicity of cisplatin and camptothecin in human brain tumor cell lines," Experimental Cell Research, vol. 243, no. 1, pp. 29-38, 1998.

[16] S. Y. Lee, "Temozolomide resistance in glioblastoma multiforme," Genes and Diseases, vol. 3, no. 3, pp. 198-210, 2016.
[17] A. Blasina, B. D. Price, G. A. Turenne, and C. H. McGowan, "Caffeine inhibits the checkpoint kinase ATM," Current Biology, vol. 9, no. 19, pp. 1135-1138, 1999.

[18] I. S. Smirnova, N. D. Aksenov, E. V. Kashuba, P. Payakurel, V. V. Grabovetsky, A. D. Zaberezhny et al., "Hepatitis C virus core protein transforms murine fibroblasts by promoting genomic instability," Cellular Oncology, vol. 28, no. 4, pp. 177-190, 2006.

[19] H. Vakifahmetoglu, M. Olsson, and B. Zhivotovsky, "Death through a tragedy: mitotic catastrophe," Cell Death \& Differentiation, vol. 15, no. 7, pp. 1153-1162, 2008.

[20] A. R. Kadia and G. B. Shah, "Cisplatin resistance reversal by disulfiram and caffeine," Journal of Pharmacology and Pharmacotherapeutics, vol. 7, no. 3, pp. 139-141, 2016.

[21] Y. Kawano, M. Nagata, T. Kohno et al., "Caffeine increases the antitumor effect of cisplatin in human hepatocellular carcinoma cells," Biological \& Pharmaceutical Bulletin, vol. 35, no. 3, pp. 400-407, 2012.

[22] D. Rybaczek and M. Kowalewicz-Kulbat, "Premature chromosome condensation induced by caffeine, 2-aminopurine, staurosporine and sodium metavanadate in S-phase arrested HeLa cells is associated with a decrease in Chk1 phosphorylation, formation of phospho-H2AX and minor cytoskeletal rearrangements," Histochemistry and Cell Biology, vol. 135, no. 3, pp. 263-280, 2011.

[23] E. S. Newlands, M. F. G. Stevens, S. R. Wedge, R. T. Wheelhouse, and C. Brock, "Temozolomide: a review of its discovery, chemical properties, pre-clinical development and clinical trials," Cancer Treatment Reviews, vol. 23, no. 1, pp. 35-61, 1997.

[24] S. Sun, T. S. Wong, X. Q. Zhang et al., "Protein alterations associated with temozolomide resistance in subclones of human glioblastoma cell lines," Journal of Neuro-Oncology, vol. 107, no. 1, pp. 89-100, 2012.

[25] A. Maréchal and L. Zou, "DNA damage sensing by the ATM and ATR kinases," Cold Spring Harbor Perspectives in Biology, vol. 5, no. 9, 2013

[26] E. Crescenzi, G. Palumbo, J. De Boer, and H. J. M. Brady, "Ataxia telangiectasia mutated and p21CIP1 modulate cell survival of drug-induced senescent tumor cells: Implications for chemotherapy," Clinical Cancer Research, vol. 14, no. 6, pp. 18771887, 2008.

[27] A. Nadkarni, M. Shrivastav, A. C. Mladek et al., "ATM inhibitor KU-55933 increases the TMZ responsiveness of only inherently TMZ sensitive GBM cells," Journal of Neuro-Oncology, vol. 110, no. 3, pp. 349-357, 2012.

[28] J. N. Sarkaria, E. C. Busby, R. S. Tibbetts et al., "Inhibition of ATM and ATR kinase activities by the radiosensitizing agent, caffeine," Cancer Research, vol. 59, no. 17, pp. 4375-4382, 1999.

[29] P. A. J. Muller and K. H. Vousden, "P53 mutations in cancer," Nature Cell Biology, vol. 15, no. 1, pp. 2-8, 2013.

[30] H. Hasegawa, Y. Yamada, H. Iha et al., "Activation of p53 by Nutlin-3a, an antagonist of MDM2, induces apoptosis and cellular senescence in adult T-cell leukemia cells," Leukemia, vol. 23, no. 11, pp. 2090-2101, 2009.

[31] S. Martin, H. Janouskova, and M. Dontenwill, "Integrins and p53 pathways in glioblastoma resistance to temozolomide," Frontiers in Oncology, vol. 2, 2012.

[32] M. D. Blough, D. C. Beauchamp, M. R. Westgate, J. J. Kelly, and J. G. Cairncross, "Effect of aberrant p53 function on temozolomide sensitivity of glioma cell lines and brain tumor initiating cells from glioblastoma," Journal of Neuro-Oncology, vol. 102, no. 1, pp. 1-7, 2011. 
[33] E. B. Dinca, K. V. Lu, J. N. Sarkaria et al., "p53 small-molecule inhibitor enhances temozolomide cytotoxic activity against intracranial glioblastoma xenografts," Cancer Research, vol. 68, no. 24, pp. 10034-10039, 2008.

[34] M. M. Mc Gee, “Targeting the Mitotic Catastrophe Signaling Pathway in Cancer," Mediators of Inflammation, vol. 2015, Article ID 146282, 2015.

[35] S. Mansilla, W. Priebe, and J. Portugal, "Mitotic catastrophe results in cell death by caspase-dependent and caspaseindependent mechanisms," Cell Cycle, vol. 5, no. 1, pp. 53-60, 2006.

[36] A. Skwarska, E. Augustin, and J. Konopa, "Sequential induction of mitotic catastrophe followed by apoptosis in human leukemia MOLT4 cells by imidazoacridinone C-1311," Apoptosis, vol. 12, no. 12, pp. 2245-2257, 2007.

[37] H. Tauchi and S. Sawada, "Analysis of mitotic cell death caused by radiation in mouse leukaemia 15178y cells: Apoptosis is the ultimate form of cell death following mitotic failure," International Journal of Radiation Biology, vol. 65, no. 4, pp. 449-455, 1994.

[38] M. Fragkos and P. Beard, "Mitotic catastrophe occurs in the absence of apoptosis in p53-null cells with a defective G1 checkpoint," PLoS ONE, vol. 6, no. 8, Article ID e22946, 2011.

[39] A. C. Ruth and I. B. Roninson, "Effects of the multidrug transporter P-glycoprotein on cellular responses to ionizing radiation," Cancer Research, vol. 60, no. 10, pp. 2576-2578, 2000.

[40] S. Hall, S. Anoopkumar-Dukie, G. D. Grant et al., "Modulation of chemotherapy-induced cytotoxicity in SH-SY5Y neuroblastoma cells by caffeine and chlorogenic acid," Toxicology Mechanisms and Methods, vol. 27, no. 5, pp. 363-369, 2017.

[41] C. R. Boeck, V. B. Marques, S. S. Valvassori et al., "Early long-term exposure with caffeine induces cross-sensitization to methylphenidate with involvement of DARPP-32 in adulthood of rats," Neurochemistry International, vol. 55, no. 5, pp. 318-322, 2009.

[42] I. V. Sorokina, T. V. Denisenko, G. Imreh, V. Gogvadze, and B. Zhivotovsky, "Reactive oxygen species regulate a balance between mitotic catastrophe and apoptosis," The International Journal of Biochemistry \& Cell Biology, vol. 81, pp. 133-136, 2016. 


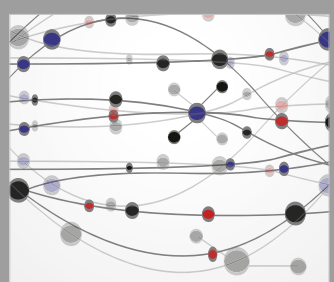

The Scientific World Journal
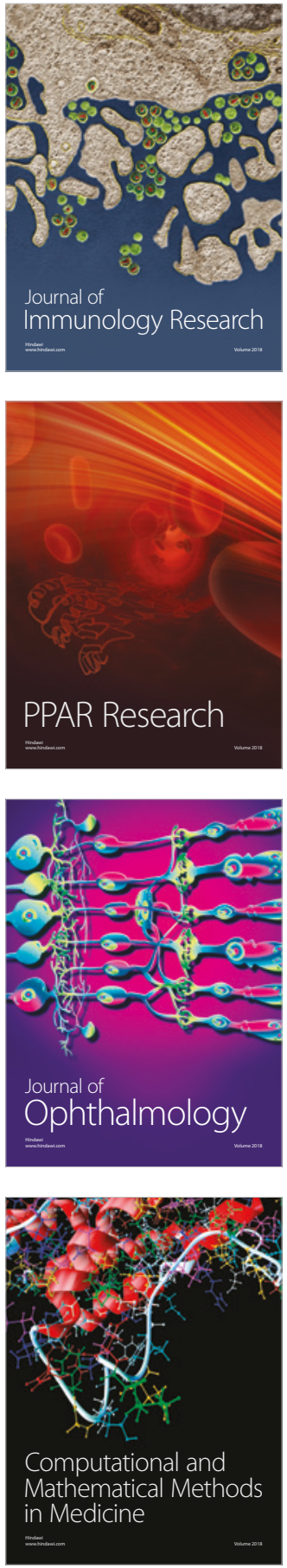

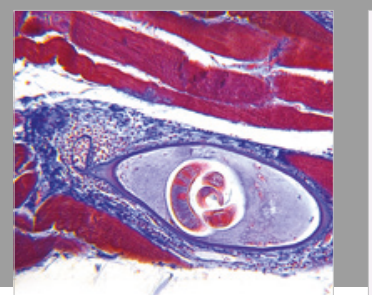

Gastroenterology Research and Practice

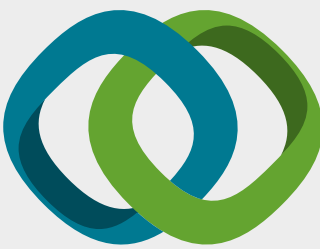

\section{Hindawi}

Submit your manuscripts at

www.hindawi.com
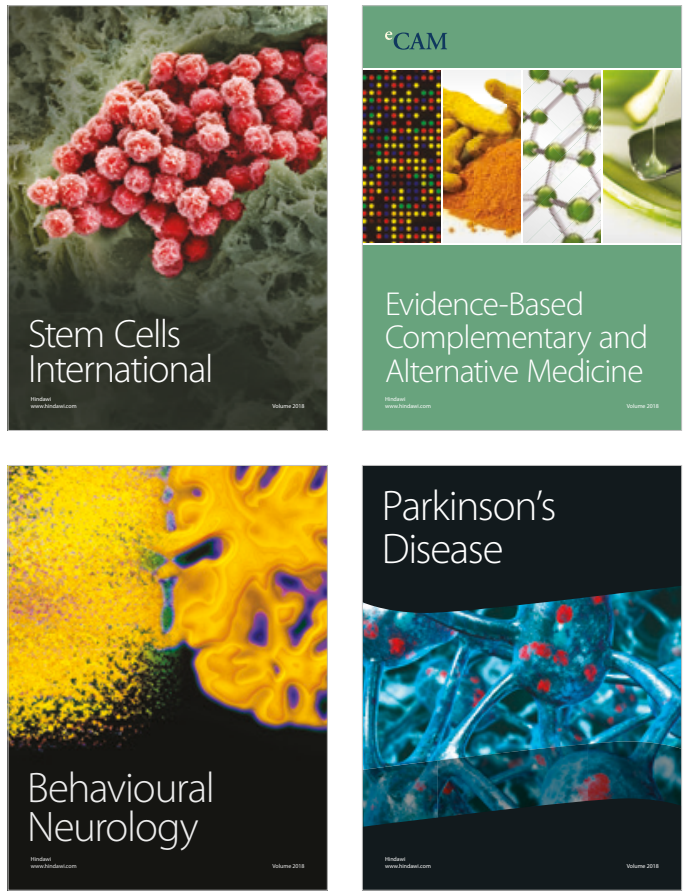

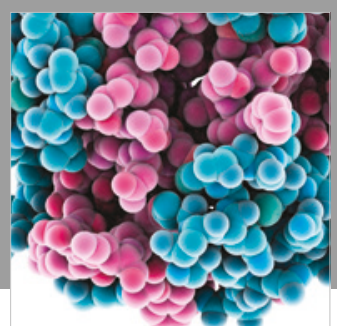

ournal of

Diabetes Research

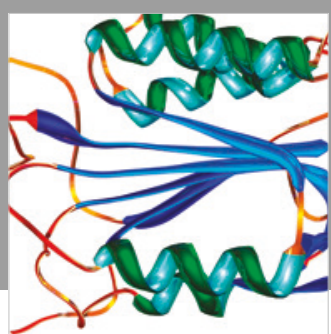

Disease Markers
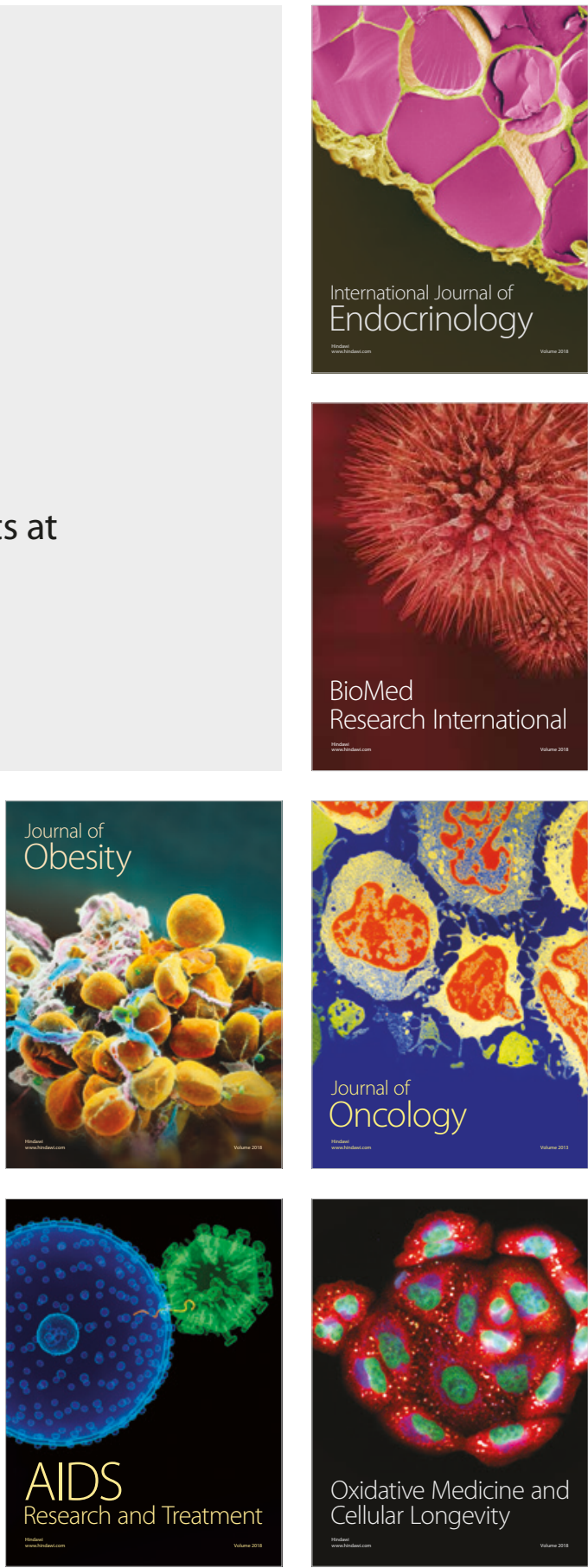